\title{
Note de synthèse sur le cycle évolutif des Sarcosporidies affectant les animaux domestiques
}

\author{
par Ch. PERROTIN $\left({ }^{*}\right)$ et M. GRABER (*)
}

\begin{abstract}
RÉSUMÉ
Après un bref rappel de la variété des espèces affectées, les auteurs rappellent la nature coccidienne des sarcosporidies. Ils exposent le cycle évolutif de ces parasites tel qu'il a pu être établi expérimentalement à ce jour. Ils mentionnent également les caractères partıculiers de ces sporozoaires, qui les différencient de Toxoplasma gondii et Hammondia hammondi.
\end{abstract}

Les sarcosporidioses (premier cas décrit par MIESCHER en 1843) sont des affections parasitaires dues à des protozoaires, se traduisant par la présence de kystes dans le tissu musculaire.

Ces affections atteignent de nombreuses espèces animales parmi les vertébrés domestiques ou sauvages.

Les sarcosporidies ont été décrites à de nombreuses reprises depuis le début du siècle, mais leur cycle évolutif était jusqu'à présent resté inconnu.

En effet, depuis quelques années seulement, faisant suite à la découverte du cycle évolutif de Toxoplasma gondii, divers auteurs ont montré la nature coccidienne des sarcosporidies, et ont en partie élucidé le cycle de développement.

Notre but ici n'est pas de faire une analyse exhaustive des travaux publiés à ce jour, mals de faire le point sur les connaissances actuelles.

\section{ESPẼCES AFFECTÉES ET RÉPARTITION GÉOGRAPHIQLE}

Les sarcosporidies sont mises en évidence à l'intérieur de formations kystiques, localısées essentiellement aux muscles striés de nombreux vertébrés.

(*) Ecole Nationale Vétérinaire de Lyon, Marcy l'Etoile, 69260 Charbonnières-les-Bains, France.
Ces formations ont été décrites chez:

- Les poissons ;

- Les reptiles (13);

- Les oiseaux (13);

- Les mammifères;

- Homme.

- Mammifères domestiques : bovins, ovins, caprins, équidés, camélidés (chameau, alpaca), chien, chat (rarement).

- Mammifères sauvages : marsupiaux, cerf, élans, gazelles, antilopes, cob defassa, zèbre, sanglier, lion, singes, etc..

Les sarcosporidioses sont cosmopolites et existent sur des aires géographiques et climatiques très différentes, comme en témoigne la variété des espèces affectées.

\section{FORMATIONS SARCOSPORIDIENNES CHEZ LES ANIMAUX DOMESTIQUES}

Les kystes sarcosporidiens se localisent aux muscles (muscles striés, cœur, œsophage, principalement), sous forme de masses plus ou moins fusiformes, de coloration blanchâtre, macro- ou microscopiques. La taille, variable en fonction de l'espèce sarcosporidienne et de l'hôte, est en moyenne de $5 \mathrm{~mm}$ de longueur sur $2 \mathrm{~mm}$ de largeur. Ces kystes sont situés à l'intérieur de la 
fibre musculaire. Ils sont limités par une membrane unique, d'épaisseur non uniforme $(25 \mathrm{~nm}$ en moyenne) (15).

Dans les kystes âgés, cette membrane délimite à l'intérieur de ceux-ci des logettes de forme variable selon l'espèce en cause.

Ces kystes renferment 2 types d'éléments parasitaires, les uns sont globuleux (12-14 $\mu$ sur 5-6 $\mu$ ) (9), et se situent à la périphérie du kyste. Ils se multiplient par endodyogénie et sont appelés métrocytes. Après plusieurs divisions, ces métrocytes vont donner naissance aux bradyzoïtes (corpuscules de Rainey).

Les bradyzoïtes, en forme de banane, ont une taille variable qui est fonction de l'espèce de sarcosporidie. PORCHET-HÉNNÉRÉ (20) montre que l'ultrastructure de ces bradyzoïtes est caractéristique de celle des germes infectieux «d'origine interne » (voir schéma $\mathrm{n}^{\circ} 1$, planche 1 ).
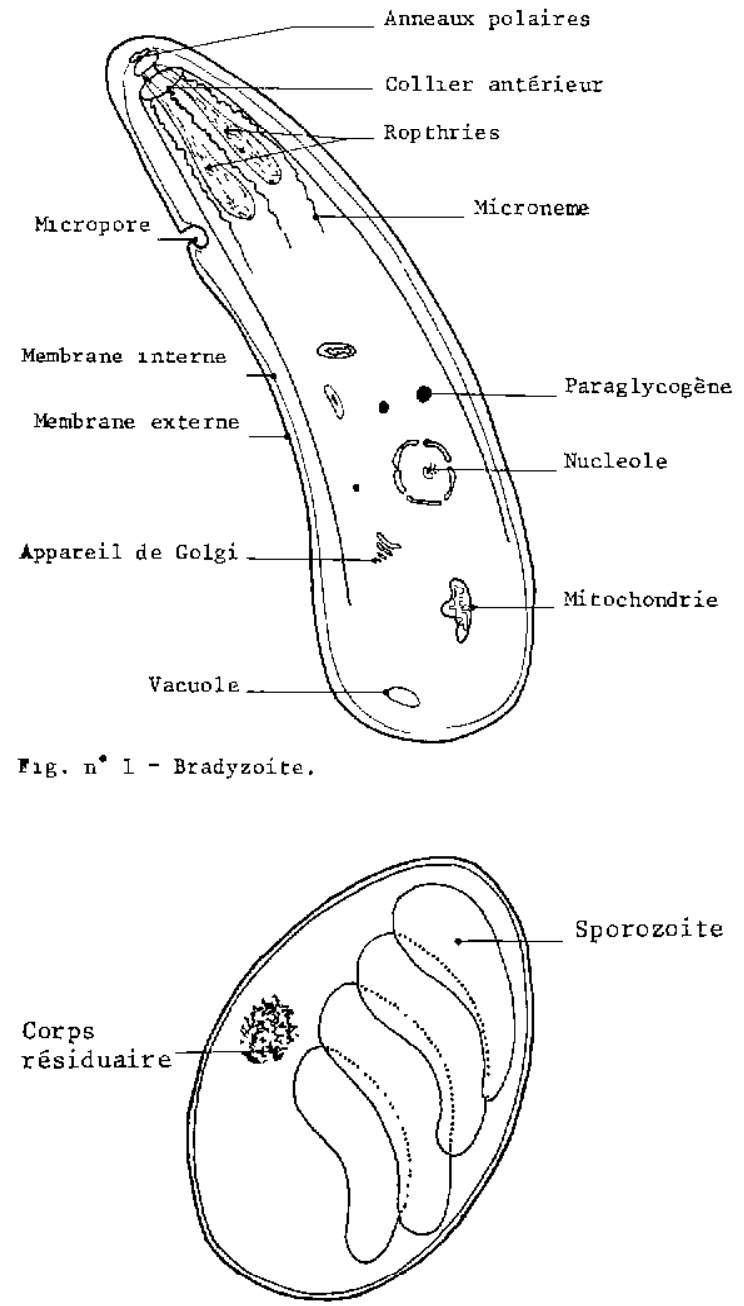

Fig. $n^{\circ} 2$ - Sporacyste de type sarcosporidien.

\section{CYCLE EVOLUTIF \\ DES SARCOSPORIDIES : DONNÉES RÉCENTES}

\section{A. Nature coccidienne des sarcosporidies}

La nature coccidienne des sarcosporidies ne fait actuellement plus aucun doute. Les infestations expérimentales de carnivores (chien, chat), réalisées à partir des kystes de Sarcocystis hirsuta (Moulé 1888) (bovins) $(8,11)$, ou de Sarcocystis tenella (Raillet 1886) (ovins) $(16,23)$, sont suivies de l'élimination avec les matières fécales d'ookystes sporulés, à paroi mince et fragile, ou le plus souvent de sporocystes isolés du genre Isospora. Le genre Isospora est caractérisé par la présence dans l'ookyste sporulé de 2 sporocystes contenant chacun 4 sporozoïtes. Ces ookystes sont différents de ceux du toxoplasme (3), et sont identiques à la grande forme de Isospora bigemina.

Ces formes sont éliminées de 10 à 20 jours en moyenne après l'infestation expérimentale. Les différents stades évolutifs du cycle coccidien (microgamètes, macrogamètes, ookystes) à l'exception de la schizogonie, sont mis en évidence dans la paroi intestinale des carnivores réceptifs $(4,8)$.

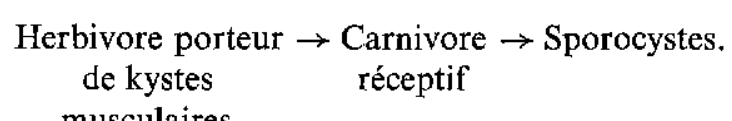
musculaires.

\section{B. Spécificité des sarcosporidies}

La mise en évidence de la nature coccidienne des sarcosporidies engendre divers travaux qui révèlent la contamination possible des herbivores à partir des sporocystes excrétés par les carnivores $(5,12,15,17)$.

Les infestations expérimentales faites avec des sporocystes de $S$. tenella (mouton), S. hirsuta (bovins), S. miescheriana (Kühn 1865) (porc), n'est possible que dans certains cas $(6,18,19,22$, 23). En effet, la réceptivité est variable selon l'espèce animale contaminée. Une espèce carnivore infestée expérimentalement peut ne pas éliminer de sporocystes, alors qu'avec le même matériel infestant, une autre espèce éliminera des sporocystes.

A ce propos, MUNDAY et RICKARD (19) observent que $S$. tenella peut se présenter dans la viande de mouton sous forme de micro- ou de 
macrokystes. Ils montrent expérimentalement que l'infestation du chien est possible avec les macrokystes, et que le cycle complet peut s'effectuer.

Macrokystes dans le $\longrightarrow$ Infection du chien tissu musculaire du mouton.<smiles>[Te][Te]</smiles>

Infection du mouton par voie buccale.

Elimination de sporocystes dans les fèces.

De la même manière, ils montrent que le cycle complet peut s'effectuer chez le chat infecté par les microkystes.

Microkystes dans le tissu musculaire Infection du chat du mouton.

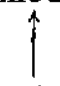

Infection du mouton par voie buccale. diaire (H. I.) et d'hôte définitif (H. D.) que LEVINE (14), compte tenu des règles internationales de nomenclature zoologique, a récemment modifiée (tabl, 1).

\section{Description détaillée du cycle évolutif tel qu'il a été établi expérimentalement}

L'hôte intermédiaire ingère des sporocystes rejetés dans le milieu extérieur avec les fèces de l'hôte définitif (carnivore ou omnivore). Les sporozoïtes libérés dans l'intestin de l'hôte intermédiaire passent dans la circulation sanguine et envahissent divers tissus $(5,12)$, y compris l'encéphale (17). La phase de multiplication asexuée (Schizogonie) se produit et conduit à la formation de kystes intramusculaires. Les schizontes sont observables en premier lieu dans les endothéliums artériels (17), puis dans les autres tissus (intestin, cæcum, foie, pancréas, rate, reins, glandes surrénales, testicules, vessie, diaphragme)(5). Ces schizontes, après un temps d'évolution, vont donner des bradyzoïtes infectants.

BERGMANN et KINDER (2) notent une différence ultrastructurale entre macro- et microkystes, confirmant ainsi les résultats expérimentaux d'infestation.

Des résultats analogues sont obtenus avec S. hirsuta.

Ces travaux révèlent que chaque espèce de Sarcosporidie ayant fait l'objet d'études expérimentales est spécifique de deux espèces ou groupe d'espèces animales :

- Un carnivore ou omnivore (hôte définitif) pour la forme coccidienne, ce qui n'est pas surprenant si l'on considère la grande spécificité des coccidies en général ;

- Un herbivore (hôte intermédiaire) chez lequel se développe le kyste.
Carnivore ou omnivore<smiles>[Te][Te]</smiles>

Tissu musculaire
Sporocystes

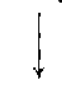

Herbivore
La nomenclature utilisée jusqu'à ces dernières années pour les sarcosporidies s'avère donc insuffisante. HEYDORN, ROMMEL et GESTRICH (10) proposent une nouvelle nomenclature faisant intervenir la notion d'hôte intermé-
Les kystes musculaires se forment en un temps variable (de 40 à 50 jours en moyenne). A ce stade, les schizontes ont disparu des autres tissus cités précédemment.

L'hôte définitif s'infecte en ingérant les kystes. Les bradyzoïtes libérés dans le tube digestif pénètrent dans la paroi intestinale et produisent directement les gamètes, sans phase de multiplication asexuée préalable $(4,17)$. Après fécondation, un ookyste se forme et sporule dans la paroi même de l'intestin. Il est ensuite éliminé dans le milieu extérieur avec les fèces. L'élimination se poursuit durant un temps variable, puis cesse spontanément.

Les sporocystes résultant d'infestations expérimentales contiennent quatre sporozoïtes et un corps résiduaire plus ou moins volumineux. Ils ne possèdent pas de micropyle, ni de corps de Stiedae. Leurs dimensions varient en fonction de l'espèce :

- Sarcocystis cruzi : $14-17 \times 9-13 \quad \mu$ (moyenne, 16,3 × 10,8 $\mu$ ).

- Sarcocystis hirsuta: $11-14 \times 7-9 \quad \mu$ (moyenne, 12,5 × 7,8 $\mu$ ).

- Sarcocystis hominis : $13-17 \times 8-11 \mu$ (moyenne, 14,7 $\times 9,3 \mu$ ). 
TABL. No I - Principales sarcosporidies de l'homme et des animaux de boucherie.

\begin{tabular}{|c|c|c|c|}
\hline Es p èces & Synonymes & H.I. & H.D. \\
\hline $\begin{array}{l}\text { Sarcocystis cruai } \\
\text { (Hasselmann, 1926), } \\
\text { Weny on, } 1926\end{array}$ & $\begin{array}{l}\text { S. fusiformis "pro parte" } \\
\text { S. marconi } \\
\text { S. bovicanis }\end{array}$ & Bovin & Divers canidés \\
\hline $\begin{array}{l}\text { Sarcooustis hirsuta } \\
\text { Moulê, } 1888\end{array}$ & $\begin{array}{l}\text { S. blanchardi } \\
\text { S. fuiformis "pro parte" } \\
\text { S. marconi } \\
\text { S. bovifetia }\end{array}$ & Bovin & $\begin{array}{l}\text { Chats domestiques } \\
\text { et sauvages }\end{array}$ \\
\hline $\begin{array}{l}\text { Sarcocystis hominis } \\
\text { Railliet et Lucet, } 1891\end{array}$ & $\begin{array}{l}\text { S. fusiformis "pro parte" } \\
\text { s. bovihominis } \\
\text { Luce vinc hominis "pro partel }\end{array}$ & Bovin & $\begin{array}{l}\text { Horme } \\
\text { Singes }\end{array}$ \\
\hline $\begin{array}{l}\text { Saroocystis civiconis } \\
\text { Heydorn, Gestrich, } \\
\text { Mehlnorn et Romme1,1975 }\end{array}$ & 5. tenella "pro parte" & Mouton & Chien. \\
\hline $\begin{array}{l}\text { Sarcocystis tenetia } \\
\text { Railliet, } 1886\end{array}$ & $\begin{array}{l}\text { Batbicona gigantea } \\
\text { S. ovifeitis }\end{array}$ & Mouton & Chat \\
\hline $\begin{array}{l}\text { Sarcocystis bertrami } \\
\text { Doflein, } 1901\end{array}$ & $\begin{array}{l}\text { S. equiconis } \\
\text { Hoarchosporidium pezlerowi }\end{array}$ & $\begin{array}{l}\text { Ane } \\
\text { Cheval }\end{array}$ & Chien \\
\hline $\begin{array}{l}\text { Sareocystis porcifetis } \\
\text { Dubey, } 1976\end{array}$ & & Porc & Chat \\
\hline $\begin{array}{l}\text { Sarcomstis nieschemiono } \\
\text { Kühn, } 1865\end{array}$ & 5. porahominis & Porc & Honme \\
\hline
\end{tabular}

- Sarcocystis ovicanis : $13-16 \times 8,5-11 \mu$ (moyenne, 14,8 × 9,9 $\mu$ ).

- Sarcocystis tenella : $11-14 \times 8-9 \quad \mu$ (moyenne, $12,4 \times 8,1 \mu$ ).

- Sarcocystis bertrami : $15-16 \times 9-11 \quad \mu$ (moyenne, 15,2 × $10 \mu$ ).

- Sarcocystis mieschieriana: $12,6 \times 9,6 \mu$.

- Sarcocystis porcifelis : $13-11 \times 7-8 \mu$.

\section{DISCUSSION}

A. Il semble que le cycle évolutif des sarcosporidies nécessite obligatoirement l'intervention de deux hôtes. En effet, les tentatives faites pour montrer l'existence d'un cycle coccidien banal chez l'hôte définitif se sont avérées infructueuses $(4,7,22)$. L'autoinfestation paraît impossible ; il n'y aurait donc pas pour les sarcosporidies l'équivalent du cycle court direct rencontré chez le toxoplasme.
L'infestation transplacentaire, jusqu'à plus ample informé n'existe pas chez les sarcosporidies (21).

B. Si le mode de transmission des sarcosporidies, tel qu'il a été décrit précédemment est indéniable, il convient cependant de remarquer que l'hôte définitif peut, dans certains cas, être porteur de kystes musculaires. C'est le cas pour :

- l'homme, chez qui vingt-et-un cas de sarcosporidiose ont été décrits entre 1868 et 1965. Le parasite reçoit le nom de Sarcocystis lindemani (Lindeman 1863), ou de Sarcocystis hominis (Rosenberg 1892);

- le chien et le chat, cas décrits par KRAUSE (1863).

Il semble que dans les cas présents, le type d'évolution pourrait être voisin de celui décrit dans la toxoplasmose du chat.

C. Il semble également, en l'état actuel des connaissances, que la transmission directe soit impossible. Cependant, AWAD (1) décrit chez 
le mouton la possibilité d'une transmission de la sarcosporidiose dans cette espèce à partir « d'éléments " présents dans les fèces.

D. Nous l'avons vu, les sarcosporidies diffèrent du toxoplasme par l'impossibilité d'un cycle coccidien banal chez l'hôte définitif. Elles diffèrent également de Hammondia hammondi, coccidie du chat, dont le cycle évolutif nécessite obligatoirement deux hôtes (chatsouris), mais chez qui une phase de schizogonie se produit dans l'intestin de l'hôte définitif.

\section{CONCLUSION}

L'originalité du cycle évolutif des sarcosporidies, réside dans le fait que 2 hôtes sont obligatoires pour sa réalisation.

Les sarcosporidies sont différentes du toxoplasme et de Hammondia hammondi. Les formes libres dans le milieu extérieur sont assimilées à l'espèce Isospora bigemina grande forme, ce qui pose le problème d'une nomenclature plus précise pour les coccidies des carnivores.

\section{SUMMARY}

Review on sarcosporidia life cycle in domestic animals

After a brief recalling of various infected species, the author remind the coccidian nature of sarcosporidia. The author describe the experimental life cycle of those parasits. The author mention the differential characters with Toxoplasma gondii and Hammondia hammondi.

\section{RESUMEN}

Nota de sintesis sobre el ciclo evolutivo de los Sarcosporidios en los animales domésticos

Después de una breve recordación de la variedad de las especies afectadas, los autores recuerdan la naturaleza coccidiana de los Sarcosporidios. Exponen el ciclo evolutivo de dichos parasitos tal como pudo establecerse experimentalmente hasta ahora. Mencionan tambien los carácteres particulares de estos esporozoarios que los diferencian de Toxoplasma gondii y de Hammondia hammondi.

\section{BIBLIOGRAPHIE SOMMAIRE (*)}

1. AWAD (F. I.). The transmission of Sarcocystis tenella in sheep. Zentbl. Bakt. ParasitKde., 1973, 42 (1) : 43-48.

2. BERGMANN (V.), KINDER (E.). Diflerences in cyst wall structure of sarcocysts in sheep. Wh. Vet. Med., 1975, 30 (20) : 772-774.

3. EUZEBY (J.), LESTRA (T.), GAUTHEY (M.). Note de recherches sur les affinités taxonomiques des Sarcosporidies. Bull. Soc. Sci. vét. Méd. comp. Lion, 1972, 74 (2) : 207-211.

4. FAYER (R.). Development of Sarcocystis fusiformis in the small intestine of the dog. Parasifology, 1974, 60 (4) : 660-665.

5. FAYER (R.), JOHNSON (A, J.) Sarcocystis fusiformis : development of cysts in calves infected with sporocysts from dogs. Proc, helminth. Soc. Wash., 1974, 41 (2) : 105-108.

6. FAYER (R.), JOHNSON (A. J.), HILDEBRANDT (P. K.). Oral infection of mammals with Sarcecystis fusiformis bradyzortes from cattle and sporocysts from dogs and coyotes. Parasitology, 1976, 62 (1) : 10-14.

(*) Une bibliographie beaucoup plus complète ( 45 références récentes) est à la disposition des lecteurs intéressés qui pourront l'obtenir gratuitement en s'adressant au centre de Documentation de l'L. E. M. V.T., 10, rue Pierre-Curie, 94700 Maisons-Alfort, France.
7. FISCHLE (B.). Investigation of the possibility o sporocysts excretion in cats and dogs given Sarcocystis tenella and Sarcocystis fusiformis sporocysts in their food. Inaugural Dissertation, Frei Universität, Berlın, 1973, 27 p.

8. GESTRICH (R.), MEHLHORN (H.), BAYSU (N.). The life cycle of Sarcosporidia. VI. Differenciation of various species of Sarcocystis fusiformis and Sarcocystis tenella. Berl. Münch. tierärztl. Wschr., 1975, 88 (10) : 191-197 et (11) : 201-204.

9. GESTRICH (R.), MEHLHORN (H.), HEYDORN (A. O.). Light and electron microscope studies on cysts of Sarcocystis fusiformis in the muscles of calves infected experimentally with oocysts and sporocysts of the large form of Isospora bigemina from cats. Zentbl. Bakt. ParasitKde, 1975, 233 A (2) : 261-276.

10. HEYDORN (A. O.), GESTRICH (R.), MEHLHORN (H.), ROMMEL (M.). Proposal for a new nomenclature of the Sarcosporidia. Zentbl. Bakt. ParasitKde, 1975, 48 (2) : 426.

11. HEYDORN (A. O.), ROMMEL (M.). The life cycle of Sarcosporidia. II. - Dogs and cats as transmitters of bovine Sarcosporidia. Berl. Münch. tierärztl. Wschr., 1972, 85 (7) : 121-123.

12. JOHNSON (A. J.), HILDEBRANDT (P. K.), FAYER (R.). Experimentally induced Sarcocystis infection in calves : pathology. Am. J. vet. Res. 1975, 36 (7) : 995-999.

13. KALGACIN (V. N.), ZASUKIN (D. M.). Distribution of Sarcocystis (Metazoa: Sporozoa) in 
vertebrates. Folia Parasit., Praha, 1975, 22 (4): 289-307.

14. LEVINE (N. D.). Nomenclature of Sarcocystis in the ox and sheep and fecal Coccidia of the dog and cat. Parasitology, 1977, 63 (1) : 36-51.

15. MEHLHORN (H.), HEYDORN, (A. O), GES TRICH (R.). Light and electron microscope studies on cysts of Sarcocystis ovicanis Heydorn and al., 1975 within muscle fibres of sheep. Zentbl. Bakt. ParasitKde, 1975, 48 (2) : 82-93.

16. MEHLHORN (H.), SCHOLTYSECK (E.), SENAUD (J.). Transmission of Sarcocystis tenella in the cat using intramuscular cysts from sheep : light and electron microscopy of the oocysts and sporocysts. C. r. hebd. Séanc. Acad. Sci., Paris, 1974, 278 (8) : 1111-1114.

17. MUNDAY (B. L.), BAKER (I, K.), RICKARD (M. D.). The developmental cycle of a species of Sarcocystis occurring in dogs and sheep, with observations on pathogenicity in the intermediate host. Zentbl. Bakt. ParasitKde, 1975, 46 (2) :111-123.

18. MUNDAY (B. L.), CORBOULD (A.). The possible role of the dog in the epidemiology of ovine sarc $)$ sporidiosis. Br. vet. $J ., 1974,130$ (1), ix-xi.

19. MUNDAY (B. L.), RICKARD (M. D.). Is Sarcocystis tenella two species? Aust. vet. J., 1974, 50 (12): 558-559.

20. PORCHET-HENNERE (E.). Ultrastructure of Sarcocystis tenella. 1. - The endozoite (after negative staining). J. Protozool., 1975, 22 (2) : 214-220.

21. ROMMEL (M.), GEISEL (O.). Prevalence and life cycle of a Sarcocystis species of the horse (Sarcocystis equicanis). Berl. Münch.. tierärztl. Wschr., 1975, 88 (24) : 468-471.

22. ROMMEL (M.), HEYDORN (A. O.), FISCHLE (B.), GESTRICH (R.). The life cycle of Sarcosporidia. V. - Additional final hosts for the Sarcosporidia of cattle, sheep and pigs and the significance of the intermediate host in the epidemiology of this parasitic disease. Berl. Münch. tierärztl. Wschr., 1974, 87 (20) : 392-396.

23. ROMMEL (M.), HEYDORN (A. O.), GRUBER (F.). Contribution to the life cycle of the Sarcosporidia. I. - The sporocysts of Sarcocystis tenella in the faeces of the cat. Berl. Münch. tierärztl. Wschr, 1972, 85 (6) : 101-105. 